\title{
CORRECTION OF FLOTATION COEFFICIENTS, DERIVED FROM ULTRACENTRIFUGATION, FOR PRESSURE AND CONCENTRATION
}

\author{
J. W. A. van den Berg and P. Le Grand \\ Twente University of Technology, Departments of Chemical Technology and of Applied Mathematics, \\ P.O. Box 217, 7500 AE Enschede, The Netherlands
}

(Received 7 April 1981)

\begin{abstract}
Flotation coefficients are usually determined from data obtained under high pressure (cell bottom) conditions. A power series expansion is proposed for correction to atmospheric pressure (meniscus) conditions. The same expansion may be applied to the concentration correction of flotation coefficients.
\end{abstract}

From sedimentation experiments with polymer solutions in the analytical ultracentrifuge, we can derive sedimentation coefficients of the polymeric component. A widely applied procedure is to identify the sedimentation coefficient with the slope of the best straight line through the data points $\ln x^{*}$ and $2 \omega^{2} t$ (where $\omega=$ angular speed in radians per second, $t=$ time in sec, $x^{*}=\left(r^{*} / r_{m}\right)^{2}, r^{*}=$ distance of the top of the Schlieren peak to the centre of rotation, $r_{m}=$ distance of the meniscus to the centre of rotation).

This procedure leads to sedimentation coefficients which depend on the concentration, and are not corrected to the pressure at the meniscus (where $p=1 \mathrm{~atm}$.). We will denote such an uncorrected sedimentation coefficient by $s$. Several procedures have been developed to obtain "true" sedimentation coefficients, corrected for pressure and concentration [1-4]. These procedures apply to dilute solutions.

When the solvent is heavier than the polymer, flotation occurs. Now the boundary layer travels from the high pressure region (where the pressure is of the order of $100 \mathrm{~atm}$.) at the cell's bottom towards the low pressure region near the meniscus. The procedures for correction for pressure which apply to sedimentation do not hold for flotation. The reason is that corrections for pressure have to be carried out for a region in the cell where the boundary layer has not proceeded "too far" away from its starting point (the meniscus, for sedimentation; the bottom for flotation). This is connected with the requirement that the time parameter $\tau \equiv 2 s_{0} \omega^{2} t$ (where $s_{0}$ is the "true" sedimentation coefficient) should not be "too far" from zero [1]. As for $\tau \rightarrow 0$ in the flotation case, the boundary is still in the high pressure region; in contrast with the sedimentation case, we cannot use the correction procedures of sedimentation for flotation without appropriate modification.

As far as we are aware, no proposals for the correction of flotation coefficients (needed for a study on permeability [6]) have been published. Pouyet and Dayantis [5] suppose for their semi-dilute solutions (concentration $>$ say $2 \%$ ) that the pressure effect affects all flotation coefficients to the same extent, i.e. as if all the concentrations were multiplied by the same (compression) factor. In a plot of $\log |s|$ vs $\log c$ this would result in a shift to the right of the whole plot, without any influence on the slope of the straight line which is characteristic for the dependence of log $|s|$ on $\log c$ in the semi-dilute region. In this paper we consider in more detail if the latter conclusions can be justified by a less qualitative reasoning. We start from the correction procedure of Dishon et al. [1] for sedimentation coefficients obtained in dilute solution.

Dishon et al. [1] wrote the solution of the (diffusion free) differential equation describing the sedimentation process (the so-called Lamm equation) as a series expansion in powers of the time parameter $\tau$. It holds only for $\tau \rightarrow 0$ :

$$
\ln x^{*}=\tau /(\alpha+1)-\frac{(2 \alpha+1) m-x}{2(\alpha+1)^{3}} \tau^{2}+\mathrm{O}\left(\tau^{3}\right)
$$

where

$$
\begin{aligned}
\tau & =2 \omega^{2} s_{0} t \\
x & =k_{s} c\left(k_{\mathrm{s}}=\text { concentration effect parameter }\right) \\
m & =\text { pressure effect parameter. }
\end{aligned}
$$

(We have not written out the clumsy third power term in full; it should not be neglected however.)

The essential point in the derivation of (1) is the assumption

$$
\begin{aligned}
& s=s(\mathrm{p}, c)=\frac{s_{0}}{\left(1+k_{s} c\right)\left(1-m+m x^{*}\right)} \\
&=\frac{s(p, 0)}{1+k_{\mathrm{s}} c}=\frac{s(1, c)}{1-m+m x^{*}}
\end{aligned}
$$

where the effects of pressure and concentration are separated; $s(p, 0)$ being the pressure dependent sedimentation coefficient corrected to zero concentration, and $s(1, c)$ being concentration dependent, but corrected to meniscus conditions were $p=1 \mathrm{~atm}$.

From equation (1) we can see that correction for pressure and concentration can be carried out independently. An easy correction for pressure for instance is to identify $s(1, c)$ with the initial slope of the plot of $\ln x^{*}$ vs $2 \omega^{2} t$. The thus obtained $s(1, c)$ can be corrected further for the effect of concentration by 
plotting $1 / s(1, c)$ vs $c$. The intercept is $1 / s_{0}$ and from the slope we can calculate the concentration dependence parameter $k_{s}$ :

$$
1 / s(1, c)=\left(1 / s_{0}\right)\left(1+k_{\mathrm{s}} c\right)
$$

A more precise procedure would be to calculate the coefficients of a cubic polynomial in $\left(2 \omega^{2} t\right)$ by a least squares approximation; the time $t$ should be taken with respect to a starting time $t_{0}$. A good approximation for $t_{0}$ is the abcis intercept of the straight line through $\left(r^{*}-r_{m}\right)$ plotted vs $t_{i}(=$ the time of the $i$-th photographed Schlieren peak with respect to the moment when the first peak was photographed).

We applied the method of Dishon et al., with the assumption (2), to flotation essentially by writing the value of their function

$\mathrm{h}\left(x^{*}\right) \equiv x^{*} /\left(1-m+m x^{*}\right)$ at $\tau=0$ not as 1 , but as, $\mathrm{h}_{\mathrm{\tau}=0}=\mathrm{q} /(1-m+m \mathrm{q})$, where $\mathrm{q}=\left(r_{\mathrm{b}} / r_{m}\right)^{2} ; r_{b}$ is the distance of the bottom of the cell to the centre of rotation. Furthermore, we introduced the distance parameter

$$
x^{\prime}=\left(r^{*} / r_{\mathrm{b}}\right)^{2}=x^{*} / \mathrm{q} .
$$

Thus, we derived as the flotation equivalent of (1) (also for $\tau \rightarrow 0$ ):

$$
\begin{aligned}
\ln x^{\prime}= & \frac{h^{2}}{\mathrm{~g}(\mathrm{~h}+x)} \tau+\frac{\mathrm{h}^{3}\left(2 \alpha \mathrm{h}_{1}+\mathrm{hh}_{1}-\mathrm{h}^{2}-\alpha \mathrm{h}\right)}{2 \mathrm{q}^{2}(\mathrm{~h}+x)^{3}} \tau^{2} \\
& +\mathrm{O}\left(\tau^{3}\right)
\end{aligned}
$$

where

$\mathrm{h}=\mathrm{h}_{\mathrm{t}=0}=\mathrm{q} /(1-m+m \mathrm{q})$

$\mathrm{h}_{1}=\mathrm{q}(1-m) \mathrm{h}_{1}=\frac{\mathrm{q}(1-m)}{(1-m+m \mathrm{q})^{2}} \cdot(1-m+m \mathrm{q})^{2}$.

Again, we have omitted the clumsy third power term; note that insertion of $q=1$ in (4) gives (1), as required.

The expansion (4) does not allow $x$ to be determined independently of $m$, the slope $s_{i}$ of the initial tangent to the plot of In $x^{\prime}$ vs $2 \omega^{2} t$ must according to (4) be written as

$$
s_{i}=\frac{s_{0}}{\left(1+\frac{x}{h}\right)} \frac{\mathrm{h}}{\mathrm{q}} .
$$

Thus, flotation coefficients corrected for pressure, but still dependent on concentration, are not obtained from the initial slopes (as was the case for sedimentation).

The conclusions of Pouyet and Dayantis [5] that in the semi-dilute region the slope of the $\log |s|-\log c$ plot is not affected by the pressure effect now may be justified by extrapolating: (a) the validity of the assumption (2) and the Lamm equation to the semidilute region, and (b) the validity of Eqns (4) and (5) to the semi-dilute region (provided that for all experiments at all concentrations $q$ has the same value, that $m$ does not depend on concentration, and that also $x / h$ is nearly constant). Typical values of $m$ and $q$ are 1.0 and $(7.2 / 5.9)^{2}=1.5$. respectively. This leads for $x=0$ to $s_{0}=1.5 \mathrm{~s}$. a much larger pressure effect cor- rection to $s$ than the corrections, obtained usually for sedimentation coefficients.

In order to obtain an idea of the order of magnitude for the correction of a flotation coefficient of a real system, we also supposed $\alpha$ to be zero for the most dilute concentration. Then, we calculated $m$ and $s_{0}$ from the first and the second coefficient of the cubic polynomial through $\ln x^{\prime}$ and $\left(2 \omega^{2} t\right)$. Then we plotted the reciprocals of the first coefficients of the other concentrations vs $c$ and calculated $k_{s}$ and the true flotation coefficient $s_{0}$ from:

$$
\begin{aligned}
& k_{\mathrm{s}}=\frac{\mathrm{q}}{1+m(\mathrm{q}-1)} \cdot \frac{\text { (slope) }}{(\text { intercept })} \\
& \left.s_{0}=\{1+m(\mathrm{q}-1)\} / \text { intercept }\right) .
\end{aligned}
$$

The use of this procedure presupposes that $m$ has been determined accurately enough for the most dilute solution, and that it remains constant over the whole concentration region. As we were only interested in an order of magnitude of the corrections for $s$ and $k_{s}$, we did not construct a more accurate iterative procedure. Experimental details have been given [6].

For the flotating system poly(2,6-dimethyl-1,4-phenylene oxide) of $\overline{\mathbf{M}}_{w} 87,000$ in trichloroethylene at $25^{\circ}$, we thus calculated $s(p, 0)=2.29$ (Svedberg) and $s_{0}=2.70$, with $m=0.5$. For the same system at $35^{\circ}$ we found $s(\mathrm{p}, 0)=2.58$ and $s_{0}=3.20$, with $m=1.1$. At both temperatures, the correction for pressure adds about $20 \%$ to $s(\mathrm{p}, 0)$. The difference found for $m$ is not a consequence of the temperature difference, but of the poor reproducibility of the assessment of the first and the second coefficients of Eqn (3).

The values obtained for this system for $m$ are in accordance with values for $m$, determined by others for other polymers in chlorinated hydrocarbon solvents [4]. From equation (1) we found for the above mentioned polymer in toluene solution at $25^{\circ}$ (where sedimentation occurs) $s(\mathrm{p}, 0)=3.12$ (Svedberg) and $s_{\mathrm{o}}=3.23$, with $m=0.7$ (in agreement with findings for other polymers in toluene $[2,3]$ ).

For the flotation case we found $k_{\mathrm{s}}=117(\mathrm{ml} / \mathrm{g})$ at $25^{\circ}$ by means of the procedure outlined above, whereas for uncorrected $s$ it was $111(\mathrm{ml} / \mathrm{g})$. This difference is not significant, however; it depends strongly on the experimental uncertainty in $m$ [cf. Eqn (6)]. Thus, at $35^{\circ}$ we found $k_{s}=89(\mathrm{ml} / \mathrm{g})$ from Eqn (6) and $k_{\mathrm{s}}=118(\mathrm{ml} / \mathrm{g})$ from uncorrected $s$ values.

\section{REFERENCES}

1. M. Dishon. M. T. Stroot, G. H. Weiss and D. A. Yphantis, J. Polym. Sci. Part A-2 8, 2163 (1970); ibid. 9, 939 (1971).

2. T. Kotaka and N. Donkai, J. Polym. Sci. Part A-2 6, 1457 (1968).

3. I. H. Billick, J. phis. Chem. 66, 1941 (1962).

4. W. J. Closs, B. R. Jennings and H. G. Jerrard, Eur. Polym. J. 4, 639, 651 (1968).

5. G. Pouyet and J. Dayantis, Macromolecules 12, 293 (1979).

6. J. W. A. van den Berg, G. van de Ridder and C. A. Smolders, Eur. Polym. J, In press. 